$\xi=$ 离

\title{
Severe Ulcerative Colitis Flare Unmasking Factor VII Deficiency
}

\author{
Paul Muna-Aguon MD ${ }^{1 *}$, Tyler Aasen DO ${ }^{1}$, Kaivan Salehpour MD ${ }^{1}$, Scott Mcshane DO ${ }^{1,2}$ \\ ${ }^{1}$ Banner University Medical Center - Phoenix, Phoenix, Arizona \\ ${ }^{2}$ Lankenau Medical Center, Wynnewood, PA \\ *Corresponding author E-mail: paulaguon@gmail.com
}

\begin{abstract}
We present a 22-year-old male with severe bleeding from Ulcerative Colitis. Workup revealed anemia and elevated prothrombin time, with subsequent mixing study and clotting factor levels revealing factor VII deficiency. Bleeding disorders such as Factor VII deficiency may exacerbate the degree of hemorrhage in inflammatory bowel disease flares, making prompt recognition of underlying coagulopathies crucial.
\end{abstract}

Keywords: Gastrointestinal Bleeding; Ulcerative Colitis; Factor VII Deficiency.

\section{Introduction}

Ulcerative colitis is a recurring inflammatory disease that usually manifests itself as gastrointestinal bleeding that affects the colon in a continuous fashion, usually beginning with the rectum (Ordas et al. 2012). Though bleeding and anemia are frequently seen with the disease, coagulopathies may also be present that will complicate or exacerbate the symptoms. We present a case of severe bleeding with Ulcerative Colitis unmasking an underlying factor VII deficiency. Factor VII deficiency is a rare congenital coagulopathy that is transmitted in an autosomal recessive pattern and is characterized by epistaxis (56\%), easy bruising (48\%), gum bleeding (34\%), hemarthrosis (16\%), and gastrointestinal bleeding (14\%) being the most common symptoms seen in patients (Mariani \& Bernardi 2009).

\section{Case}

A 22-year-old male with known ulcerative colitis and autism presented with a one-day history of fevers, lower abdominal pain, and bloody diarrhea. Ulcerative colitis had been confirmed 5 months prior by endoscopy with biopsies. He denied prior bleeding events or a family history of bleeding disorders. Vital signs revealed fever to $39.4^{\circ} \mathrm{C}$, heart rate $113 \mathrm{bpm}$, blood pressure $107 \mathrm{mmHg} /$ $70 \mathrm{mmHg}$, with physical exam significant for mild bilateral lower quadrant tenderness without rebound or guarding. Rectal examination yielded small amount of visualized bright red blood. Laboratory studies showed white blood cell count of $18.0 \mathrm{~K} / \mathrm{mm}^{3}$, hemoglobin $13.8 \mathrm{~g} / \mathrm{dL}$, platelets $344 \mathrm{~K} / \mathrm{mm}^{3}$, C-reactive protein 252 $\mathrm{mg} / \mathrm{L}$, erythrocyte sedimentation rate $55 \mathrm{~mm} / \mathrm{hr}$, and prothrombin time 24.2 seconds with international normalized ratio (INR) of 2.2. Abdominal computed tomography (CT) scan with contrast showed diffuse colonic thickening.

The patient was admitted and started on intravenous steroids and empiric antibiotics for presumed flare of ulcerative colitis. Stool studies were negative for an infectious etiology of the hematochezia. The patient continued to have heavy rectal bleeding and follow-up labs 48 hours later revealed INR 2.3. He was trialed on oral vitamin $\mathrm{K} 10 \mathrm{mg}$ daily for three days without success. Over the subsequent three days, the patient continued to have bloody diarrhea with declining hemoglobin to $10.6 \mathrm{~g} / \mathrm{dL}$ and rising INR to 2.8. A mixing study was performed which revealed a pretest prothrombin time of 27.2 seconds and post-mixing prothrombin time of 14.2 seconds, suggesting no evidence of an inhibitor affecting extrinsic pathway plasma procoagulants. Specific factor activity level and testing revealed normal factor II, V, IX, and X activity but noted a markedly reduced factor VII activity of $4 \%$ (normal 55-170\%). The patient's bleeding spontaneously subsided after several days of conservative management and he did not require infusion of recombinant factor 7 or plasma products. The diagnosis of factor VII deficiency was confirmed and the patient was discharged home after five days of hospitalization when bleeding subsided.

\section{Discussion}

Factor VII deficiency is the most common of the rare congenital bleeding disorders, affecting one per 500,000 individuals and typically manifests with prolonged prothrombin time (Lapecorella \& Mariani 2008). It can be divided into Type I and Type II, which reflects quantitative and qualitative defect respectively (Sevenet et al. 2016). Bleeding in factor VII deficiency typically presents in locations more dependent on the extrinsic pathway including the brain, bowel, uterus, placenta, lungs, and heart (Lapecorella \& Mariani 2008). Transmission occurs in an autosomal recessive pattern and presenting symptoms may include epistaxis (56\%), easy bruising (48\%), gum bleeding (34\%), hemarthrosis (16\%), and gastrointestinal bleeding (14\%) (Mariani \& Bernardi 2009). Treatment is typically reserved for cases of severe hemorrhage with treatment options including fresh frozen plasma, prothrombin complex concentrate, and recombinant factor VIIa (Mariani \& Bernardi 2009).

Ulcerative colitis is a recurring inflammatory disease that affects the colon in a continuous fashion, beginning in the rectum (Ordas et al. 2012). Inflammatory bowel disease (IBD) in the setting of a 
concomitant bleeding disorder represents a rare phenomenon that may manifest clinically in profuse gastrointestinal bleeding (Freeney et al. 1998). Severe bleeding in an IBD patient should prompt clinicians to pursue laboratory studies including a coagulation panel. If abnormal, clinicians should consider an underlying bleeding disorder. We present a rare case of severe bleeding in ulcerative colitis unmasking an underlying Factor VII deficiency. Factor VII deficiency may exacerbate the degree of hemorrhage in ulcerative colitis flares, making clinical suspicion and prompt recognition of underlying coagulopathies crucial.

\section{References}

[1] Ordas I, Eckmann L, Talamini M, Baumgart DC, Sandborn WJ Ulcerative Colitis. Lancet 2012; 380: 1606-1619. https://doi.org/ 10.1016/S0140-6736(12)60150-0.

[2] Mariani G, Bernardi F. Factor VII Deficiency. Seminars in Thrombosis and Hemostasis 2009; 35: 400-406. https://doi.org/10.1055/s0029-1225762.

[3] Lapecorella M, Mariani G. Factor VII deficiency: defining the clinical picture and optimizing therapeutic options. Haemophilia 2008; 14(6): 1170-1175. https://doi.org/10.1111/j.13652516.2008.01844.

[4] Sevenet P, Kaczor DA, Depasse F. Factor VII Deficiency: From Basics to Clinical Laboratory Diagnosis and Patient Management. Clin Appl Thromb Hemost 2016. https://doi.org/10.1177/107 6029616670257.

[5] Freeney M, Talbot R, Worsley A, Snook J. Crohn's disease and inherited coagulopathy: two case reports. Ital J Gastroenterol Hepatol 1998; 30(4): 442. 